\title{
Mechanical vibrations of pendant liquid droplets
}

\author{
Robert H. Temperton, Michael I. Smith, and James S. Sharp ${ }^{a}$
}

School of Physics and Astronomy and Nottingham Nanotechnology and Nanoscience Centre, University of Nottingham, University Park, Nottingham, UK, NG7 2RD

Received: date / Revised version: date

\begin{abstract}
A simple optical deflection technique was used to monitor the vibrations of microlitre pendant droplets of deuterium oxide, formamide, and 1,1,2,2-tetrabromoethane. Droplets of different volumes of each liquid were suspended from the end of a microlitre pipette and vibrated using a small puff of nitrogen gas. A laser was passed through the droplets and the scattered light was collected using a photodiode. Vibration of the droplets resulted in the motion of the scattered beam and time dependent intensity variations were recorded using the photodiode. These time dependent variations were Fourier transformed and the frequencies and widths of the mechanical droplet resonances were extracted. A simple model of vibrations in pendant/sessile drops was used to relate these parameters to the surface tension, density and viscosity of the liquid droplets. The surface tension values obtained from this method were found to be in good agreement with results obtained using the standard pendant drop technique. Damping of capillary waves on pendant drops was shown to be similar to that observed for deep liquid baths and the kinematic viscosities obtained were in agreement with literature values for all three liquids studied.
\end{abstract}

\section{Introduction}

Vibrations of liquid droplets are important for a number of applications including droplet atomisation [1], small scale mixing/demixing [2] and the actuation/ratcheting of liquid drops on surfaces [3-6]. There has also been an interest in the use of droplet vibrations as a tool for measuring the material properties of liquids [7-10] and viscoelastic mate-

${ }^{a}$ Corresponding author: james.sharp@nottingham.ac.uk rials [11-14]. These applications involve excitation of the vibrational modes of the droplets either by driving the droplets at their mechanical resonant frequencies $[1-3,6]$ or by applying mechanical impulses to the drops $[10,14]$. Recent experiments indicate that excitation with broadband noise sources can also be used [15].

When small amplitudes of oscillation are excited in liquid drops, their vibrational modes can be interpreted by 
relatively simple models and theories which consider how capillary-gravity waves propagate along the surface of the droplets. The first theory of droplet vibrations was developed by Lord Rayleigh [16] for spherical inviscid liquid globules i.e. freely suspended (or levitated) droplets with zero viscosity. This important work predicted that the mechanical vibrational frequencies, $f$, of freely suspended liquid droplets depend upon their radius $R$, density, $\rho$, and surface tension $\gamma$ according to the relation

$$
f=\sqrt{\left(\frac{n(n-1)(n+2) \gamma}{4 \pi^{2} \rho R^{3}}\right)}
$$

where $n=2,3,4 \ldots$ is a mode number. This theory was later extended by Chandrasehkar [17] to include liquids of small but finite viscosity. He showed that in this limit, the vibrational frequencies are similar to those observed for inviscid droplets. He also showed that the full width at half maximum, $\Delta f$, (hereafter referred to as the width) of the mechanical resonance peaks depends upon the viscosity, $\eta$, of the droplets according to

$$
\Delta f \sim \frac{\eta}{\rho R^{2}}
$$

These results have been subsequently confirmed in experiments designed to study the vibration of magnetically [10] and acoustically [18] levitated droplets.

A more convenient geometry for generating and studying droplet vibrations involves the use of sessile (substrate supported) or pendant (hanging) drops. The reason for this is that the methods used to levitate droplets often require specialised equipment which generates large magnetic or acoustic fields. In comparison, a droplet can be deposited on a surface or suspended from the end of a nozzle/tube and vibrated with relative ease. However, there are additional considerations when interpreting the vibrational properties of sessile and pendant drops. For example, the presence of a substrate can have a significant influence on the damping of the droplet motion and on the permissible vibrational modes [19].

Pendant drops in particular are routinely used to measure the surface tension of liquids, although this is not usually done using vibrational methods. Instead, the pendant drop technique [20] is based around analysing high resolution images of drops that have been allowed to deform under the influence of gravity. Droplets with sizes larger than the capillary length of the fluid $\left(L_{c}=\sqrt{\frac{\gamma}{\rho g}}\right.$, where $g$ is the acceleration due to gravity) will deform under their own weight. During this deformation, the droplets extend and their surface area increases. Surface tension acts to oppose the deformation until the change in gravitational potential energy balances the change in interfacial energy. Analysis of the resulting drop shapes allows the surface tension of the liquid to be estimated [20].

A major limitation of the pendant drop technique is that it only works when the droplets are larger than the capillary length of the fluid. Alternative techniques must therefore be found if the surface tension of submillitre droplets need to be determined. One such approach involves measurement of their vibrational response. The present study considers a low cost optical deflection based approach to the measurement of these vibrational properties. A vibrational approach such as this could easily be 
extended to high throughput applications in microfluidics applications.

While there is some theoretical work examining the oscillation of pendant drops [7], there has been relatively little experimental work done in the area. In contrast, there have been numerous studies that provide models that attempt to describe the vibrational response of sessile drops [19,21-26]. Perhaps the most simple and intuitive of these models was derived by Noblin et al. [23] who modelled droplet oscillations as standing waves on a liquid bath of finite depth. This approach has since been used by authors including Sharp et. al who applied the model to study how the fundamental vibrational mode of sessile drops varied with the three phase contact angle [24] and viscosity of the liquid [19]. More recently, Temperton and Sharp extended the model in an attempt to describe the vibrational properties of droplets with an asymmetric shape that were formed by placing droplets on a patterned surface [25].

Noblin's approach considers the surface of the drop as a bath of liquid of finite depth, $h$, on which standing capillary waves can occur. In the case where the three phase contact line of the drop is fixed, the permissible wave vectors, $k$, for these states are given by the condition that a half integer number of wavelengths fit along the circumferential profile length, $l$ of the drop (see figure 1d). This gives the result that $k=\frac{n \pi}{l}$, where $n$ is an integer (mode number). Inserting this result into the dispersion relation for capillary-gravity waves leads to the following equation for the resonant frequencies of vibration, $f$, of the drop. [23]

$$
f^{2}=\left(\frac{n g}{4 \pi l}+\frac{n^{3} \pi \gamma}{4 \rho l^{3}}\right) \tanh \left(\frac{n \pi h}{l}\right)
$$

where $\gamma$ and $\rho$ are the surface tension and density of the liquid respectively.

This study aims to show that the same analysis can be applied to the study of pendant drops of three different liquids. A key part of this work involves extending the studies of Noblin et al. [23] and Sharp et al.[19, 24,25] and to include experimental studies and a discussion of how damping effects influence the vibrational properties of pendant liquid drops with sizes smaller than the capillary length of the liquids being studied.

\section{Experimental}

Deuterium oxide $\left(\mathrm{D}_{2} \mathrm{O}\right)$, formamide, and 1,1,2,2-tetrabromoethane were obtained from Sigma Aldrich and were used as received. These liquids were chosen because when used in combination they allow the influence of surface tension and viscosity on drop vibration to be probed independently. For example, $\mathrm{D}_{2} \mathrm{O}$ and formamide have similar value of the ratio $\frac{\gamma}{\rho}$, but different values of kinematic vis$\operatorname{cosity}\left(\frac{\eta}{\rho}\right)$, while formamide and 1,1,2,2-tetrabromoethane have similar kinematic viscosities but different values of the ratio $\frac{\gamma}{\rho}$. Disposable neoprene gloves were worn during the handling of the liquids.

Microlitre droplets of these liquids were suspended from the end of a clean microlitre pipette (see figure 1a). Light from a HeNe laser $(\lambda=633 \mathrm{~nm}, 1 \mathrm{~mW}$, Research ElectroOptics) was passed through the drops and allowed to fall 
a)
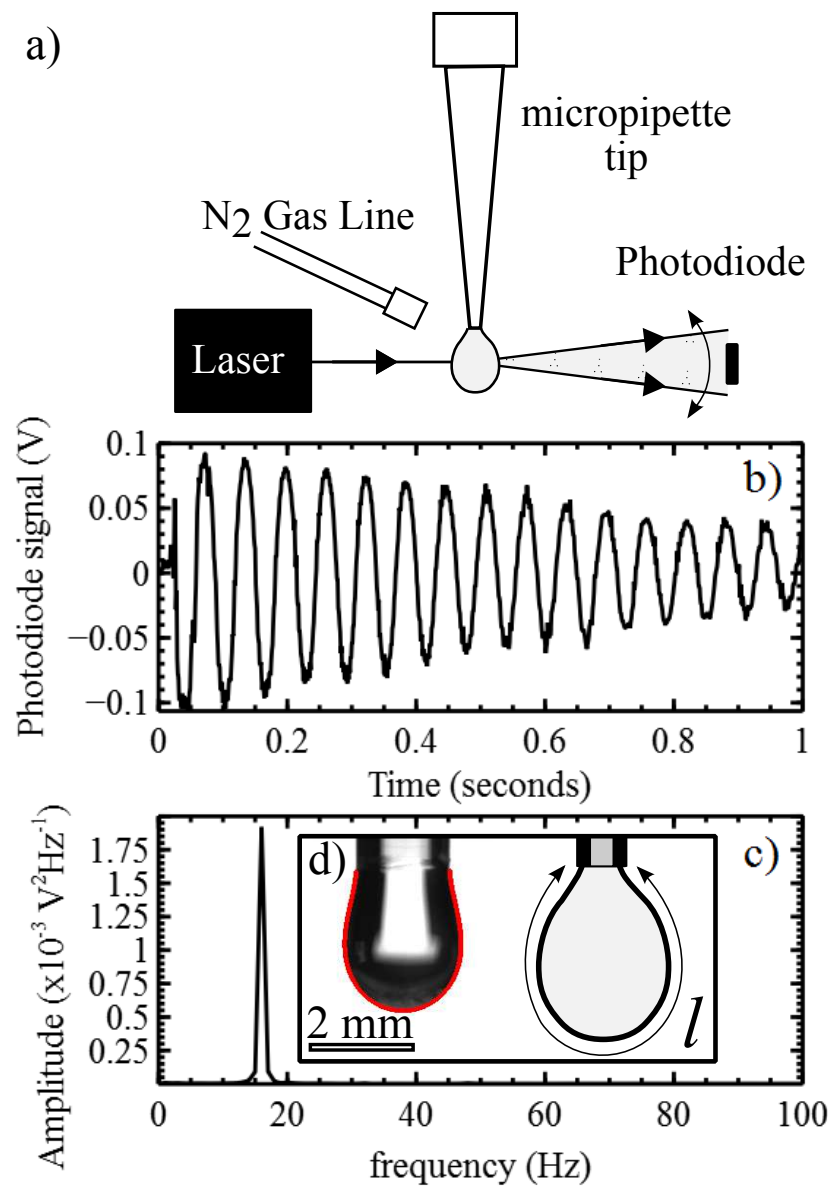

Fig. 1. Experimental setup. a) Droplets were suspended from the end of a micropipette tip and vibrated using a short puff of nitrogen gas. b) Time dependent variations in the intensity of light refracted through a $4 \mu l$ drop of 1,1,2,2-tetrabromoethane provides information about its vibrational response. c) Fourier transformation of the intensity signal in part b) gives the mechanical vibrational spectrum. d) Images of the drop were used to extract the circumferential profile length, $l$. The profile of larger drops were fitted to the form of the pendant drop equations [20] and used to extract an independent measure of the surface tension of the liquids being studied (see text). on the surface of a silicon photodiode which was attached to a home built amplifier circuit and connected to a computer using a National Instruments USB-6008 data acquisition card. In each case, the small liquid droplets acted like a poor lens and defocussed the laser beam to produce a diffuse round spot with an intensity distribution that decayed radially away from its centre.

An impulse was applied to the drops in the form of a short puff of nitrogen gas. This short puff was delivered to the drops via an $8 \mathrm{~mm}$ diameter plastic tube connected to a pressurised nitrogen line and actuated using an electrically controlled pneumatic valve via software written in Matlab. The puff of nitrogen was delivered from above at an angle of $\sim 45^{\circ}$ to the plane of incidence and an angle of $\sim 45^{\circ}$ to the horizontal. Other directions were also tried and no significant dependence of the vibrational properties on the excitation conditions was observed. In each case, the duration of the impulse was set to $100 \mathrm{~ms}$ and the puff of gas caused the droplet to vibrate, resulting in deflections of the scattered laser beam. The photodiode was positioned near the edge of the scattered light spot in such a way that the intensity gradients present in the spot created time dependent variations in the measured intensity when the droplets were vibrated (see figure $1 \mathrm{~b}$ ).

The resulting time dependent electrical signals obtained from the photodiode were Fourier transformed to obtain the vibrational spectrum of the liquid droplets using software written in Matlab (MathWorks). After use, the small liquid droplets were absorbed onto paper towels and disposed of using correct chemical disposal procedures. 
A typical example of the electrical signal obtained from the photodiode along with the corresponding Fourier transform is shown in figure 1 . As this figure shows, the vibration of the drop gives rise to a decaying periodic electrical signal which shows characteristics of a damped harmonic oscillator. In theory, Fourier transformation of these signals should give a series of independent peaks which correspond to the different vibrational modes of the drops. However, in reality (and as shown below), vibrational modes be given as an integral. However, the value of $h$ used in higher than the fundamental tend to be more heavily dampedthis study was calculated from real images of droplets. As As a result, they have much broader spectral widths and lower amplitudes than the fundamental mode in the vibrational spectrum. A consequence of this is that the simple optical deflection technique very often only detects the lowest frequency of vibration (as shown in figure 1). For this reason, much of the analysis that follows will concentrate on this lowest frequency mode.

The central frequencies of the peaks were found to decrease with increasing size of the droplets and to increase with increasing surface tension of the liquid being used. The full width at half maximum (hereafter referred to as the width) of the peaks was measured directly from the vibrational spectra and was found to increase with the viscosity of the liquid used and to decrease with increasing drop size.

\section{A Manta G-125B Camera (Allied Vision Technologies)} was used to obtain images of the droplets (see figure 1d). These images were used to measure the circumferential profile length, $l$, and the average depth, $h$, of each droplet.
The average depth, $h$, of the droplets was determined by using a Matlab program to generate a surface of revolution from the droplet profile (see inset figure 2) . This surface was used to work out what fraction of the total surface area, $\frac{d A}{A}$, of the drop was at a given distance, $y$, from the end of the pipette tip. The value of $h$ was then calculated as a weighted average of these values using the formula $h=\Sigma y \frac{d A}{A}$. Ideally, the definition of $h$ should such, the data was discrete and a discrete sum was used to calculate the value of $h$ in each image. In practice, this involved splitting the image of the droplet into horizontal strips that were a pixel thick and calculating the surface area of revolution $(d A)$ of each strip. The total surface area of the droplet, $A$, was then calculated by summing all the values of $d A$. The perpendicular distance, $y$, (see figure 2 inset) of the horizontal strip from the end of the pipette was determined and multiplied by the fraction of the surface area of the droplet $\left(\frac{d A}{A}\right)$ at that distance. The average depth/height of the droplet, $h$, was then calculated by summing all the $y \frac{d A}{A}$ values.

The shapes of droplets larger than the capillary length and larger than those used in the vibration experiments were also captured using the camera and fitted using the pendant drop equations [20]. This approach provided an independent measure of the surface tension of the liquids being studied. 


\section{Results and Discussion}

The top panel of figure 2 shows plots of how the square of the vibrational frequency, $f^{2}$, of the fundamental $(n=2)$ mode varies as a function of $\tanh \left(\frac{n \pi h}{l}\right) \frac{1}{l^{3}}$ for the three liquids studied. In each case, the dimensions of the drop (and hence $h$ and $l$ ) were varied and the frequency of vibration determined for each drop size. For droplets significantly smaller than the capillary length of the liquid, the first term in the brackets in equation 3 becomes negligible and such a plot should result in a straight line. Above the capillary length, the vibrational frequency is expected to become independent of the properties of the liquid as the drop motion becomes dominated by gravity. The transition to this regime is marked by a changing drop size dependence of the vibrational frequency at low values of $\tanh \left(\frac{n \pi h}{l}\right) \frac{1}{l^{3}}$ as shown in figure 2 . The inset in this figure shows the same frequency data plotted as a function of $\tanh \left(\frac{n \pi h}{l}\right) \frac{1}{l}$, which should be independent of the liquid properties for drops with sizes much larger than the capillary length (according to equation 3). This inset shows that the frequencies of vibration of the droplets start to converge as the droplets become larger.

For the liquids studied, the capillary lengths $\left(L_{c}=\sqrt{\frac{\gamma}{\rho g}}\right)$ are $2.6 \mathrm{~mm}, 2.3 \mathrm{~mm}$ and $1.3 \mathrm{~mm}$ for $D_{2} O$, formamide and 1,1,2,2-tetrabromoethane respectively. A summary of the literature values of the surface tension and density used to calculate these capillary lengths are given in table 1 . The dashed line in the inset in figure 2 marks the position of the capillary length for 1,1,2,2-tetrabromoethane in the units used on the horizontal axis of this plot. As this liq-
Table 1. Physical properties of the liquids used at $25^{\circ} \mathrm{C}$. All values were obtained from reference [27]

\begin{tabular}{lccc}
\hline \hline Liquid & $\gamma\left(\mathrm{mJm}^{-2}\right)$ & $\rho\left(\mathrm{kgm}^{-3}\right)$ & $\eta(\mathrm{mPas})$ \\
\hline $\mathrm{D}_{2} \mathrm{O}$ & 72.0 & 1107 & 1.0 \\
Formamide & 58.2 & 1134 & 3.3 \\
$1,1,2,2-$ tetra & 49.7 & 2967 & 9.7 \\
bromoethane & & & \\
\hline \hline
\end{tabular}

uid has the smallest capillary length, the dashed line is an upper bound for the crossover between the capillary and gravity dominated regimes for the three liquids studied here. In constructing this dashed line it is noted that the relevant length scale being compared to the capillary length is not the circumferential profile length $l$. A more appropriate length scale (and the one which is used here) is given by determining when the two terms in the bracket in equation 3 become comparable i.e. when $L_{c}=\frac{l}{2 \pi}$. The length scale $\frac{l}{2 \pi}$ gives an approximation to the radius of curvature and as such provides a more accurate measure of the drop dimensions.

The solid lines in figure 2 are fits to the data in the capillary dominated regime. According to equation 3, the slope of this region should be equal to $\frac{\gamma \pi n^{3}}{4 \rho}$. The range of values used in the fits in figure 2 , were for droplets that are at least a factor of $\sim 5$ smaller than the capillary lengths of the liquids studied. This condition was chosen to ensure that the data were fitted in a linear region away from the transition between the capillary and gravity dominated regimes. Values of $\frac{\gamma}{\rho}$ obtained from the fits in figure 2 are given in table 2 along with corresponding values calculated 

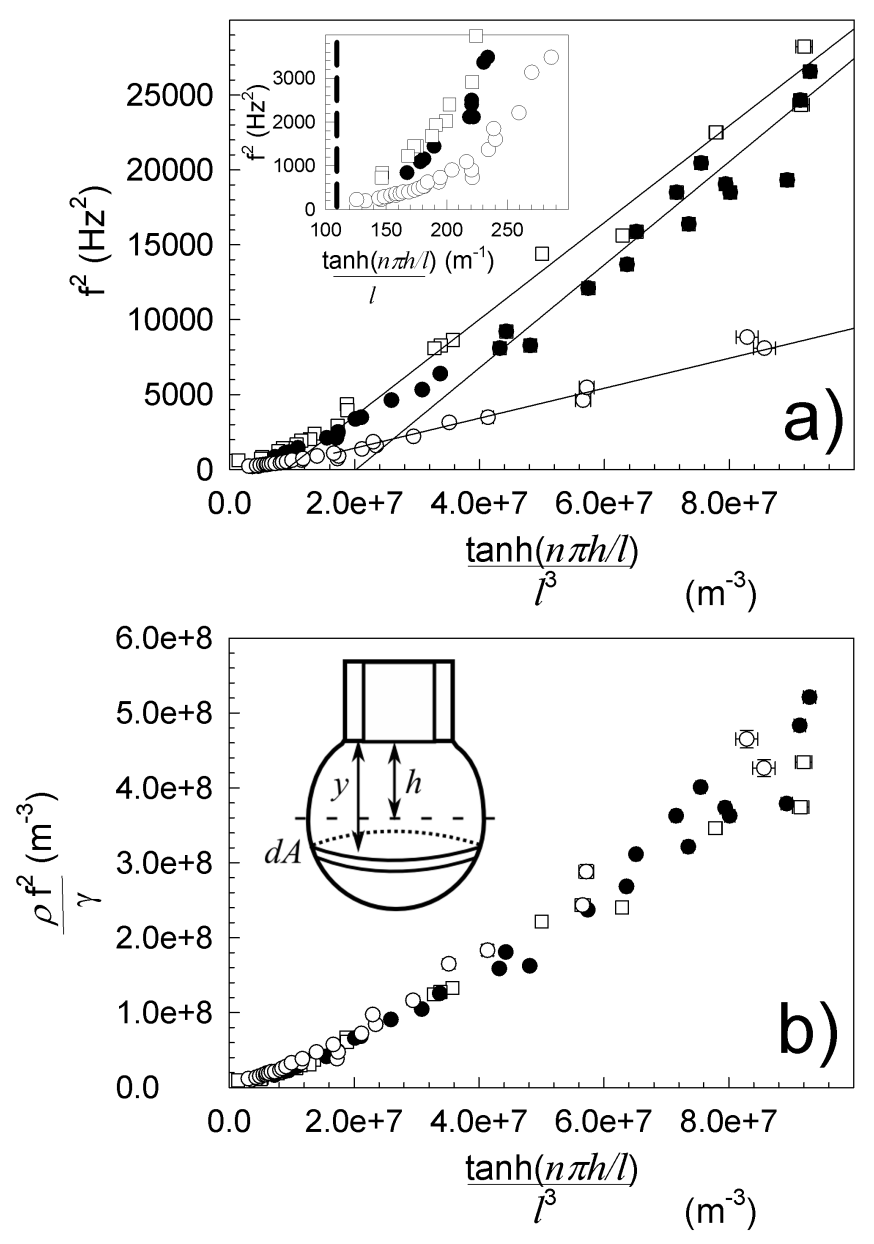

Fig. 2. Size dependence of the vibrational frequencies of $\mathrm{D}_{2} \mathrm{O}(\square)$, formamide $(\bullet)$ and 1,1,2,2-tetrabromoethane $(\bigcirc)$ droplets. Panel a) The solid lines are fits to the data for drops with radii less than one fifth of the capillary length, $L_{c}$, of each fluid. The inset in this panel shows that as the drop size increases and approaches the capillary length (marked with the dashed line), the vibrational frequency becomes independent of liquid properties and the plots start to converge (as predicted by equation 3). Panel b) shows the data in panel a) rescaled by the ratio of $\frac{\gamma}{\rho}$ using the experimentally determined values in table 2. The inset in this panel shows a diagram explaining how the area element $d A$ was defined during the calculation of $h$ (see text). from the data given in table 1 . In all cases, a value of $n=2$ was used in converting the slope of the fits to the values given in the second column of table 2 . The bottom panel of figure 2 shows all the data in the top panel rescaled by the ratio of $\frac{\gamma}{\rho}$ obtained from the fits. As this figure shows, the data in the capillary regime collapses onto a master curve, thus confirming the scaling predicted by equation 3 when the gravitational term is neglected.

Comparison of the values obtained for the ratio $\frac{\gamma}{\rho}$ in table 2 with literature values shows that the experimentally determined values are in agreement within the limits of experimental uncertainty. The only exception occurs for $\mathrm{D}_{2} \mathrm{O}$ where the experimentally determined value is lower than predicted using the literature values. The density of the $\mathrm{D}_{2} \mathrm{O}$ used here is unlikely to be greater than the literature value quoted. This suggests that the surface tension of the $\mathrm{D}_{2} \mathrm{O}$ was lower than $72 \mathrm{mJm}^{-2}$.

Contamination of the $\mathrm{D}_{2} \mathrm{O}$ surface by exposure to air could provide a possible explanation for why the surface tension might be lower than predicted. Like water, $\mathrm{D}_{2} \mathrm{O}$ has a high surface energy when compared to simple organic liquids. A $\mathrm{D}_{2} \mathrm{O}$ surface can therefore become rapidly contaminated by organic compounds in the air or possibly through contact with pipettes or other surfaces if they are not cleaned thoroughly enough. Previous work has shown that contamination of water surfaces by exposure to air can occur on the time scales of minutes [1]. This time scale is comparable to the time taken to set the experiment up and to start collecting data. 
The other liquids studied here are organic liquids, having surface energies that are much lower $\left(50-60 \mathrm{mJm}^{-2}\right.$ [27]) than that of water $/ \mathrm{D}_{2} \mathrm{O}$ and are less likely to show evidence of surface contamination effects. This may explain why the agreement between the slopes in figure 2 and the literature values is much better for these liquids than the slope obtained for $\mathrm{D}_{2} \mathrm{O}$. 
Table 2. Comparison of fitted parameters with literature values obtained from table 1

\begin{tabular}{lcccc}
\hline \hline Liquid & $\begin{array}{c}\left(\frac{\gamma}{\rho}\right)_{l i t} \\
\times 10^{-5}\left(\mathrm{~m}^{3} \mathrm{~s}^{-2}\right)\end{array}$ & $\begin{array}{c}\left(\frac{\gamma}{\rho}\right)_{f i t} \\
\times 10^{-5}\left(\mathrm{~m}^{3} \mathrm{~s}^{-2}\right)\end{array}$ & $\begin{array}{c}\left(\frac{\eta}{\rho}\right)_{l i t} \\
\times 10^{-6}\left(\mathrm{~m}^{2} \mathrm{~s}^{-1}\right)\end{array}$ & $\begin{array}{c}\left(\frac{\eta}{\rho}\right)_{f i t} \\
\end{array}$ \\
\hline $\mathrm{D}_{2} \mathrm{O}$ & 6.5 & $5.1 \pm 0.2$ & 0.9 & $1.3 \pm 0.1$ \\
Formamide & 5.2 & $5.5 \pm 0.3$ & 2.9 & $2.5 \pm 0.1$ \\
$1,1,2,2-$ tetra & 1.7 & $1.6 \pm 0.1$ & 3.3 & $2.8 \pm 0.1$ \\
bromoethane & & & & \\
\hline \hline
\end{tabular}


Assuming that the density of the $\mathrm{D}_{2} \mathrm{O}$ used in this study was equal to the literature value, the experimentally determined value of $\frac{\gamma}{\rho}$ obtained from the fits given in table 2 gives $\gamma_{D_{2} O}=(56 \pm 2) \mathrm{mJm}^{-2}$ for the surface tension of the liquid. This is comparable to the surface tension of organic liquids (as mentioned above) and would be consistent with the presence of a contaminating layer of organic material on the surface of the drops.

A simple experiment was performed to determine whether significant surface contamination of the $\mathrm{D}_{2} \mathrm{O}$ droplets had occurred. Once the vibration experiments on the $D_{2} O$ droplets had been completed, the volume of the drop was increased until its size was so large that distortions in its shape (due to gravitational effects) were significant enough to be observed by eye. An image of the large pendant $D_{2} \mathrm{O}$ drop was collected within a few seconds of expanding the drop volume. The shape of the droplet profile was then fitted using the standard pendant drop technique [20]. This simple experiment allowed the surface area of the drop to be increased and imaged on much shorter time scales than those that are associated with contamination of the liquid surface by exposure to air. The surface tension value obtained was $\gamma=(61 \pm 2) m J m^{-2}$. This value is similar to (but slightly higher) than the value obtained from the vibration experiments. It is also significantly lower than the literature value quoted in table 1 . This suggests that there may have been some contamination of the $\mathrm{D}_{2} \mathrm{O}$ liquid. Given that the liquid surface was expanded quite quickly and before significant airborne contaminants could re-pollute the surface of the drops, this would seem to in- dicate that the contaminants were already present in the liquid.

When similar (pendant drop technique) experiments were repeated for large droplets of the other two liquids studied, values of $\gamma=(60 \pm 2) \mathrm{mJm}^{-2}$ and $\gamma=(53 \pm 2) \mathrm{mJm}^{-2}$ were obtained for formamide and 1,1,2,2-tetrabromethane respectively. These are comparable to the literature values given in table 1 and to the values extracted from the fits to the vibration data shown in figure 2 and table 2 .

Regardless of the fact that there may have been some contamination of the $\mathrm{D}_{2} \mathrm{O}$ surface, it is important to point out that the analysis of the vibrational frequencies described here provides a similar value of the surface tension to that obtained using the pendant drop technique. This means that the vibrational measurements are extracting the 'correct' value for the surface tension of the contaminated $\mathrm{D}_{2} \mathrm{O}$. In fact, these results suggest that the vibrational method used here could be used to monitor/detect the presence of contaminants on liquid surfaces by monitoring changes in surface tension.

These combined results and the favourable comparison between the surface tension values obtained from the vibration experiments and pendant drop technique provide support for the simple model described here. This work suggests that the model successfully captures the physics of pendant drop vibration providing that the $n=2$ mode describes the lowest permissible vibrational mode in incompressible pendant droplets. In the case of sessile droplets, the $n=2$ mode was shown to be lowest mode for an incompressible fluid $[19,24]$ with a pinned contact 
line. The fact that gravity is not important in the capillary dominated regime means that the orientation of the drop (sessile or pendant) should not influence the physics of vibration. However, some small differences in the vibrational properties might be expected as a result of differences in the contact area between a drop and a solid substrate and a drop attached to a pipette filled with liquid. In the latter case, much of the base of the droplet will be in contact with a fluid reservoir. As such, the boundary conditions experienced by the drop are likely to differ from those where the droplet sits on a solid surface.

Given the similarities between pendant and sessile drops with dimensions below the capillary length, it is reasonable to assume that the vibrational modes available to a pendant drop are similar to those observed for sessile droplets. Based on this assumption, the lowest vibrational mode for a pendant drop should appear as a rocking/swinging motion, similar to that reported by Daniel et al [3] for sessile drops. However, to confirm that this is indeed the case, a Dalsa HC640 camera was used to obtain a movie of a vibrating pendant drop at 300 frames per second (see movie1.avi in electronic supplementary information). Figure 3 shows a frame taken during the vibration of a $\mathrm{D}_{2} \mathrm{O}$ droplet at the point where it reached its maximum vibrational amplitude. This figure also shows a diagram of the expected shape of the droplet at its maximum amplitude for the $\mathrm{n}=2$ mode. The predicted and measured shapes of the vibrating drop are in agreement, suggesting that the lowest mode of pendant drop vibration corresponds to a rocking/swinging motion analogous to that of a sessile drop.

Viscous damping of the droplet motion is also expected to influence the mechanical vibrational spectra of the droplets. Such effects show up predominantly in changes in the width of the spectra, although when damping effects become larger, some changes in the vibrational frequencies of drops can be observed [14]. Previous studies have considered the effects of viscous damping on droplet motion [1, 19]. Sources of damping include viscous dissipation effects in the liquid, which may be enhanced by the presence of a substrate, or as a result of the presence of surface contamination layers. The simplest model of damping considers viscous dissipation of energy by the capillary-gravity waves on the surface of the drops [28]. This model predicts that the width of the resonance peaks should vary according to the equation

$$
\Delta f=\frac{\eta}{\pi \rho} k^{2}=\frac{\eta \pi n^{2}}{\rho l^{2}}
$$

which has a similar functional form to equation 2 for the spectral widths of levitated droplets.

The top panel of figure 3 shows that plots of the width of the mechanical resonance against $\frac{1}{l^{2}}$ display a linear relationship over the entire range of droplet sizes studied. The solid lines in this figure represent fits to the data for the three liquids studied using equation 4 . The slopes of these fits were used to calculate an estimate of the ratio $\frac{\eta}{\rho}$ (the kinematic viscosity) and the results are summarised in table 2. The bottom panel of figure 3 shows the same width data rescaled by the experimentally de- 
termined ratios of $\frac{\eta}{\rho}$ from this table. The solid line in the bottom panel of the figure is a fit to all the data and has a slope of $12.3 \pm 0.4$. This is in agreement with the value of $4 \pi(\sim 12.6)$ predicted by equation 4 .

The values of kinematic viscosity obtained for both formamide and 1,1,2,2-tetrabromoethane are comparable to, but lower than the values obtained from the literature values in table 1 . However, these differences are small enough that they could be accounted for in terms of changes in viscosity caused by fluctuations in room temperature. The droplets were vibrated under ambient conditions and as such, their properties will have been susceptible to the ambient temperature in the room. Values of the viscosity of these liquids vary in the range $2.83-3.23 \mathrm{mPas}$ for formamide and $8.4-9.7 \mathrm{mPas}$ [29] for 1,1,2,2-tetrabromoethane [30] over the temperature range 25-30 ${ }^{\circ} \mathrm{C}$. Temperature changes could therefore account for a range of possible values for the kinematic viscosity of $2.5-2.8 \times 10^{-6} \mathrm{~m}^{2} \mathrm{~s}^{-1}$ for formamide and $2.9-3.3 \times 10^{-6}$ $m^{2} s^{-1}$ for 1,1,2,2-tetrabromoethane being observed during these experiments. A comparison shows that these ranges encompass the values obtained from the spectral width measurements for these liquids (see table 2).

In the case of $D_{2} O$, the kinematic viscosity obtained from the vibration experiments is higher than the value predicted by literature values at a temperature of $25^{\circ} \mathrm{C}$. Changes in temperature are unlikely to account for the differences in the fitted and literature values as the kinematic viscosity typically varies in the range $0.9-1.0 \times 10^{-6}$ $m^{2} s^{-1}$ for this liquid between 25 and $30^{\circ} C$ [31]. The in-
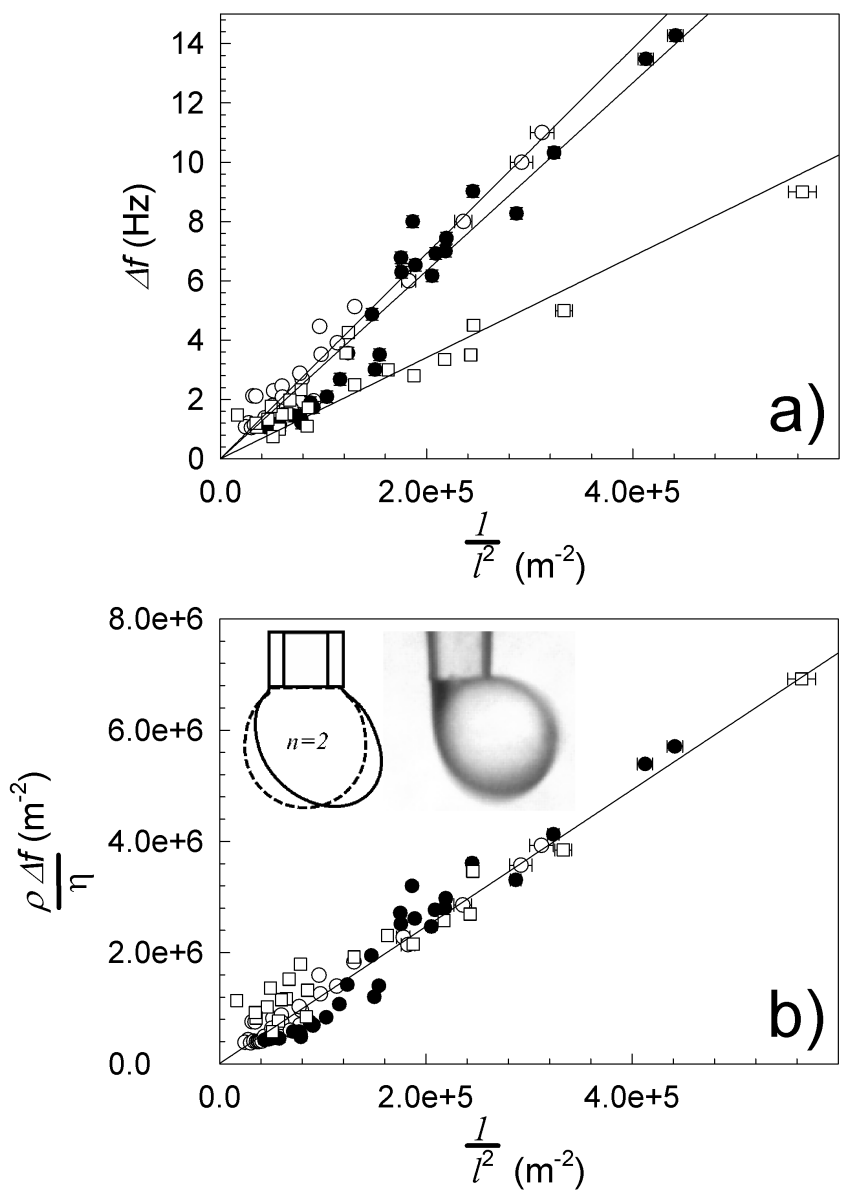

Fig. 3. Drop size dependence of the spectral width for $\mathrm{D}_{2} \mathrm{O}(\square)$, formamide $(\bullet)$ and 1,1,2,2-tetrabromoethane $(\bigcirc)$ droplets. The solid lines in panel a) are fits to the experimental data. Panel b) shows the same width data rescaled by the experimentally determined value of $\frac{\eta}{\rho}$ obtained from the fits to the data in panel a). The solid line in panel b) is a fit to all the data and has a slope of $12.3 \pm 0.4$. The inset in this panel shows a schematic diagram of the unperturbed drop shape (dashed line) and the expected shape of the $n=2$ vibrational mode. A photo is also shown of a $D_{2} O$ drop at its maximum amplitude of vibration for comparison (as obtained using a $300 \mathrm{fps}$ camera, diameter of pipette $1.7 \mathrm{~mm}$ ). 
creased kinematic viscosity in the $D_{2} \mathrm{O}$ samples is more likely to be caused by the presence of the same contaminants that were responsible for changing the surface tension of the drops. The $\mathrm{D}_{2} \mathrm{O}$ used in these experiments was freshly opened prior to the experiments and should contain minimal levels of bulk contaminants. However, contaminants could have been introduced as a result of contact with foreign surfaces. If low surface tension impurities were dissolved/suspended in the liquid as a result of this contact, they could quickly segregate to the surface and result in a significant increase in the damping of droplet vibrations $[19,32,33]$. Such an increase in damping due to surface effects would manifest as an increase in the kinematic viscosity if the data is interpreted using the simple model described here.

The apparent validity of equation 4 over the entire range of values of $l$ for all three liquids studied is quite striking. Previous work on sessile drops has shown that damping effects due to the substrate can have a significant influence on the width of the droplet mechanical resonances for small drops and small three phase contact angle values [19]. This occurs because the presence of the substrate causes the fluid velocity to decay over distances comparable to the size of the drop. These distances are typically much smaller than those observed for the decay of the velocity near the surface of an infinitely deep fluid. This creates steeper fluid velocity gradients which result in greater energy dissipation. However, this effect is only present when the drop profile is shallow and does not appear to be significant for large contact angles [19].
The pendant drops studied here have oscillatory behaviour which appear to be consistent with effects that are comparable to the damping of capillary waves on the surface of an infinitely deep liquid bath. This is quite interesting and may be due to the fact that the area of contact between the droplet and the tip of the micropipette is small in comparison to that between a sessile drop and a solid substrate.

The fact that the model used here is able to produce values of the surface tension and viscosity that are close to the literature values for three separate liquids is extremely encouraging. This work indicates that there is some value in the application of simple models (such as the one described here) to the study of vibrating liquid drops.

A recent review of drop vibration has criticised the use of simple models such as the one discussed here when trying to describe the vibration of pendant/sessile liquid droplets [26]. The simplistic approach to modelling the droplets makes use of the assumption that they can be described as a bath of liquid with an average depth. In reality, consideration should be given to the fact that each point on the drop surface will be at a different distance from the substrate. Moreover, a full solution to the drop vibration problem would include an expansion of the different surface modes of the drop, rather than the use of a simplification of the resonance condition used here. We agree with the authors of the previous review that the model described here should not be used to make detailed quantitative predictions of the oscillatory behaviour of liquid droplets. It should, at best, be used only to provide 
estimates of the vibrational properties of sessile and pendant droplets. In a recent study by Sharp et al., an even simpler version of this model was used, where these authors made the substitution $\alpha^{2}=\tanh \left(\frac{n \pi h}{l}\right)$ in equation 3. They showed that a value of $\alpha \sim 0.81$ could be used to provide an approximation to the vibrational frequencies of sessile droplets over a broad range of contact angles [24]. For spherical cap droplets with sizes below the capillary length, this approximation works because the hyperbolic tangent function in equation 3 is only weakly dependent on contact angle in the range studied. A similar approach could have also been applied to the pendant droplets studied here. However, experimentally determined values of the average height of the drop were used to calculate the hyperbolic tanh function so that a more detailed comparison of experiment and model could be performed.

\section{Conclusions}

A simple optical deflection method was used to monitor the vibrational response of microlitre droplets of $\mathrm{D}_{2} \mathrm{O}$, formamide and 1,1,2,2-tetrabromoethane. Measurements of the frequencies and spectral widths of the lowest vibrational mode were used to obtain estimates of the surface tension and kinematic viscosities of the liquids. This was done using a simple model of droplet vibration which relates these quantities to the circumferential profile length of the drops. The values obtained were found to be in agreement with literature values of these quantities and with independent measurements. Moreover, an analysis of the width of the droplet resonances suggested that the damping of capillary waves on the surface of the drops is similar to that observed on an infinitely deep liquid bath. This makes analysis of the data and extraction of the kinematic viscosity much easier than in the case of sessile droplets where substrate damping effects can dominate. The existence of bulk-like damping in the pendant droplets was attributed to a reduced contact between the drop and the micropipette when compared to a sessile drop on a surface.

\section{Acknowledgements}

JSS thanks the Leverhulme Trust (grant reference RPG2012-702) for providing financial support for this work. MIS gratefully acknowledges the support of a Royal Society University Research Fellowship and the EPSRC (UK) for funding under grant reference EP/L003651/1.

\section{Author Contribution Statement}

RHT and JSS collected the droplet vibration data and wrote the manuscript. MIS wrote the software for analysis of the large pendant drops and provided comments on the manuscript.

\section{References}

1. B. Vukasinovic, M.K. Smith and A. Glezer, J. Fluid Mech. 587, (2007) 395-423.

2. F. Mugele, J.C. Baret and D. Steinhauser, Appl. Phys. Lett. 88, (2006) 204106. 
3. S. Daniel, S. Sircar, J. Gliem, and M.K. Chaudhury, Langmuir 20, (2004) 4085-4092.

4. S. Daniel, M.K. Chaudhury and P.G. DeGennes, Langmuir 21, (2005) 4240-4248.

5. A. Shastry, M.J. Case and K.F. Bohringer, Langmuir 22, (2006) 6161-6167.

6. K.R. Langley and J.S. Sharp, Langmuir 26, (2010) 1834918356.

7. E.D. Wilkes and O.A. Basaran, Phys. Fluids 9, (1997) 15121528.

8. W. Meier, G. Greune, A. Meyboom and K.P. Hofmann, Eur. Biophys. J. 29, (2000) 113-124.

9. I. Egry, H. Giffard and S. Schneider, Meas. Sci. Tech. 16, (2005) 426-431.

10. R.J.A. Hill and L. Eaves, Phys. Rev. E 81, (2010) 056312

11. G.A. Coquin, J. Acoust. Soc. Am. 36, (1964) 1052.

12. J.C. Chrispell, R. Cortez, D.B. Khismatullin and L.J. Fauci, Physica D 240, (2011) 1593-1601.

13. H. Akimoto, K. Nagai and N. Sakurai, J. Appl. Mech. 79, (2012) 041002.

14. R.H. Temperton, R.J.A. Hill and J.S. Sharp, Soft Matter 10, (2014) 5375-5379.

15. S. Mettu and M.K. Chaudhury, Langmuir 28, (2012) $14100-14106$.

16. R.W.S. Rayleigh, Proc. R. Soc. London 29, (1879) 71-97.

17. S. Chandrasekhar, Proc. London Math. Soc. 3, (1959) 141149.

18. C.L. Shen, W.J. Xie and B. Wei, Phys. Rev. E 81, (2010) 046305.

19. J.S. Sharp, Soft Matter 8, (2012) 399-407.

20. O.I. del Rio and A.W. Neumann, J. Colloid Int. Sci. 196, (1997) 136-147.
21. M. Strani and F. Sabetta, J. Fluid Mech. 141, (1984) 233247.

22. R.W. Smithwick III and J.A.M. Boulet, J. Coll. Int. Sci. 130, (1989) 588-596.

23. X. Noblin, A. Buguin and F. Brochard-Wyart, Eur. Phys. J. Spec. Top. 166, (2009) 7-10.

24. J.S. Sharp, D.J. Farmer and J. Kelly, Langmuir 27, (2011) 9367-9371.

25. R.H. Temperton and J.S. Sharp, Langmuir 29, (2013) 4737-4742.

26. A.J.B. Milne, B. Defez, M. Cabrerizo-Vilchez and A. Amirfazli, Adv. Coll. Int. Sci. 203, (2014) 22-36.

27. R.C. Weast (Editor), Handbook of Chemistry and Physics, 69th Ed. (CRC Press, London 1989)

28. L.D. Landau and E.M. Lifshitz, Fluid Mechanics, 2nd Ed. (Elsevier, Oxford 1987) 93

29. A.M. Cases, A.C. Gomez Mrigliano, C.M. Bonatti and H. N. Solimo, J. Chem. Eng. Data 46, (2001) 712-715.

30. G.R. Cokelet, F.J. Hollander and J.H. Smith, J. Chem. Eng. Data 14, (1969) 470-473.

31. F.J. Millero, R. Dexter and E. Hoff, J. Chem. Eng. Data 16, (1971) 85-87.

32. J. Miles, J. Fluid Mech. 222, (1991) 197-205.

33. S.T. Milner, J. Fluid Mech. 225, (1991) 81-100. 